\title{
P04-28. Breadth and magnitude of neutralizing antibody responses in subjects infected with HIV-I subtype D or recombinants in Rakai district, Uganda
}

L Eller*1, B Ouma ${ }^{2}$, P Oballah², MA Eller ${ }^{1}$, MS de Souza ${ }^{3}$, F WabwireMangen ${ }^{4}$, O Laeyendecker ${ }^{5}$, T Quinn ${ }^{5}$, N Kiwanuka ${ }^{4}$, D Serwadda ${ }^{4}$, N Sewankambo 6 , M Wawer ${ }^{5}$, R Gray ${ }^{5}$, NL Michael ${ }^{1}$, ML Robb ${ }^{1}$, D Montefiori ${ }^{7}$, BK Brown ${ }^{1}$ and VR Polonis ${ }^{1}$

\begin{abstract}
Address: ${ }^{1}$ Makerere Univ. Walter Reed Project/US Military HIV Research Program, Kampala, Uganda, ${ }^{2}$ Makerere University Walter Reed Project, Kampala, Uganda, ${ }^{3}$ Armed Forces Research Institute of Medical Sciences, Bangkok, Thailand, ${ }^{4}$ Makerere Univ. Walter Reed/Makerere Univ School of Public Health, Kampala, Uganda, 5 Johns Hopkins School of Medicine, NIAID, NIH, Baltimore, MD, USA, ${ }^{6}$ Makerere University College of Health Sciences, Kampala, Uganda and ${ }^{7}$ Duke University, Durham, NC, USA

* Corresponding author
\end{abstract}

from AIDS Vaccine 2009

Paris, France. 19-22 October 2009

Published: 22 October 2009

Retrovirology 2009, 6(Suppl 3):P56 doi:10.1 186/1742-4690-6-S3-P56

This abstract is available from: http://www.retrovirology.com/content/6/S3/P56

(C) 2009 Eller et al; licensee BioMed Central Ltd.

\section{Background}

Limited information is available regarding HIV-1 neutralizing antibody (NAb) responses in clade D infection. Here we assess the breadth and magnitude of NAb responses in clade $\mathrm{D}$ or $\mathrm{D}$ recombinant infections, and correlate both to clinical outcomes.

\section{Methods}

Participants were enrolled in an HIV-1 incidence cohort in Rakai district, Uganda, between 1999 and 2004. The TZMbl NAb assay was performed against a panel of 10 Tier 2 pseudoviruses (2 each of clades A, B, C, D and CRF02 AG) using one early plasma (within 1 year of seroconversion) and one later sample (3-4 years after seroconversion) from 21 participants ( 11 Clade D and 10 recombinants). Subtype was determined using a Multi-Region Hybridization assay.

\section{Results}

Overall, subjects showed more NAb against subtypes A, AG and D, compared to subtypes B and C. Increased magnitude was observed at later timepoints in $81 \%$ (17/21) of subjects, with geometric mean titers against the 10 viruses ranging from $<20$ to 1035 . NAb breadth increased in $71 \%$ $(15 / 21)$ of subjects. No statistical differences were seen in magnitude or breadth comparing recombinant versus clade D infections. Strikingly, higher NAb magnitude in both the early $(P=0.048)$ and late $(P=0.009)$ plasmas was associated with progression to AIDS. Higher viral load (>50,000 copies/ml) at the early timepoint was associated with greater magnitude $(\mathrm{p}<0.0001)$ and breadth $(\mathrm{p}=$ 0.04 ) of neutralization over time.

\section{Conclusion}

These data demonstrate an expansion of breadth and magnitude of NAb over time in subtype $\mathrm{D}$ and $\mathrm{D}$ recombinant HIV-1 infections in Uganda, with more frequent and potent responses directed against subtypes A, CRF02 $A G$, and D. Higher viral load was associated with greater breadth and magnitude of neutralization, indicating that increased antigen load with viral replication may be driving the expansion of pseudovirus NAb responses in this cohort, with no observed benefit in clinical outcome. 